\title{
Primary cns Lymphoma: Analysis of Treatment by Gamma- Knife Radiosurgery and Chemotherapy in a Prospective, Observational Study
}

\author{
Andres Mauricio Alvarez*, Jose E Valerio, Sammie Coy, Alan A Stein BS and Aizik Wolf
}

Department of Neurological Surgery, Miami Neuroscience Center at Larkin, 6129 SW 70th St, South Miami, FL 33143, USA

\begin{abstract}
Background: Primary central nervous system lymphomas (PCNSL) include $4 \%$ of all primary brain-tumors. It can involve the brain parenchyma, leptomeninges, eyes and the spinal cord. Unlike systemic lymphoma, durable remissions remain uncommon. Different combinations of chemotherapy and Methotrexate are the gold standard. This is a clinical study comparing the outcomes using Methrotexate and Methrotexate with Gamma Knife Radiosurgery (GKRS).

Methods: Retrospective, comparative study. Study arms, control group performed by Methotrexate as a single agent, in dose of $8 \mathrm{~g} / \mathrm{m}^{2}$ and treatment group Methrotexate and GKRS. Strict inclusion and exclusion criteria were employed and review of histologically confirmed PCNSL. Primary objective were survival rate. Secondary outcomes were the remission or re-incidence of a lesion.

Results: Between June 1995 and May 2015, 128 charts were evaluated. 73 subjects in Control group and 55 in GKRS group. Follow-up period 24 to 49 months (mean 36.9 months). There were no statistically significant differences in patient demographics or histology diagnosis. GKRS doses ranging from 12 Gy to 26 Gy (median: $13 \mathrm{~Gy}$ ). Median survival rate 26.8 months control group and 47.6 in GKRS group from the $1^{\text {st }}$ pharmacological treatment. All lesions had complete response after GKRS at 3-8 weeks (mean range 5.8 weeks). Control group (820 weeks).

Conclusion: GKRS is non-invasive, safe, and shows rapid success, improving the prognosis of the patients. This noninvasive treatment modality should be considered as an alternative or co-adjuvant option in PCNSL. Level of Evidence: Retrospective, Comparative study (Level III).
\end{abstract}

Keywords: Primary CNS lymphoma; Radiosurgery; Gamma knife; Chemotherapy; Brain tumor; Methotrexate; Lymphoma

\section{Introduction}

Primary central nervous system lymphoma (PCNSL) is a rare cancer accounting for less than $4 \%$ of brain tumors [1]. These lesions usually present as intra-axial nodules with diffuse meningeal, periventricular, and perivascular spread being very common. The primary central nervous system extranodal high-grade malignant cells are usually large or immunoblastic cells of B-cell non-Hodgkin's, tracing their origin back to the brain parenchyma, spinal cord, leptomeninges or eyes and are typically limited to the central nervous system (CNS) with infrequent extension outside the nervous system. Treatment modalities for patients with newly diagnosed PCNSL include radiation and/or chemotherapy [2]. While the role of radiation therapy for initial management of PCNSL is controversial, clinical trials will attempt to improve the therapeutic index of this modality. Routes of chemotherapy administration include intravenous, intraocular, intraventricular or intra-arterial. Multiple trials have outlined different methotrexatebased chemotherapy regimens and have used local techniques to improve drug delivery. A major challenge in the management of patients with PCNSL remains the delivery of aggressive treatment with preservation of neurocognitive function $[3,4]$. Routinely PCNSL present as single or multifocal, massive infiltrative lesions that may occur in the cortex, and penetrating deep into the white and/or gray matter. The lesions may show areas of necrosis especially in patients with immunodeficiencies. GKRS has not yet been used universally in the treatment of the PCNSL and is non-invasive, safe and shows prompt and successful results, improving the prognosis and quality of life of the patient. Like other brain tumors, one of the major contests in treatment with chemotherapeutic agents remains the delivery of therapeutic concentrations of drugs to the CNS. A study of blood-brain barrier
(BBB) permeability in a rat brain tumor model established a large heterogeneity of microvascular leakage; the vasculature within and around the brain tumors has a wide range of permeability, from typical capillaries with no (BBB) leakage to a tumor vasculature that allows free entry of large molecules. Although MTX crosses the BBB, remote minus is quantifiable in the brain tissue than in the serum. High-dose $\operatorname{MTX}\left(>1 \mathrm{~g} / \mathrm{m}^{2}\right)$ has been shown to be an independent factor correlating with survival [5-8]. Thus, MTX is administered in high doses, up to $8 \mathrm{~g} / \mathrm{m}^{2}$, in order to achieve therapeutic drug concentrations in the tumor and surrounding brain. Intravenous (IV) doses less than $1 \mathrm{~g} / \mathrm{m}^{2}$, similar to what has been used in the treatment of other malignancies outside the brain, reach CNS concentrations generally felt not to be cytotoxic The purpose of this retrospective comparative study is to determine the effectiveness of chemotherapy using methotrexate in dose of $8 \mathrm{~g} / \mathrm{m}^{2}+$ radiosurgery in the treatment of PCNSL.

\section{Materials and Methods}

This is a retrospective, comparative study evaluating the effect and

*Corresponding author: Andres Mauricio Alvarez, Department of Neurological Surgery, Miami Neuroscience Center at Larkin, 6129 SW 70th St, South Miami, FL 33143, USA, Tel: (786) 871-6800; E-mail: andresmauricioalvarez07@gmail.com

Received October 13, 2015; Accepted November 08, 2015; Published November 17, 2015

Citation: Alvarez AM, Valerio JE, Coy S, Alan A Stein BS, Wolf A (2015) Primary cns Lymphoma: Analysis of Treatment by Gamma- Knife Radiosurgery and Chemotherapy in a Prospective, Observational Study. J Integr Oncol 4: 150. doi:10.4172/2329-6771.1000150

Copyright: @ 2015 Alvarez AM, et al. This is an open-access article distributed under the terms of the Creative Commons Attribution License, which permits unrestricted use, distribution, and reproduction in any medium, provided the original author and source are credited. 
Citation: Alvarez AM, Valerio JE, Coy S, Alan A Stein BS, Wolf A (2015) Primary cns Lymphoma: Analysis of Treatment by Gamma- Knife Radiosurgery and Chemotherapy in a Prospective, Observational Study. J Integr Oncol 4: 150. doi:10.4172/2329-6771.1000150

survival rate of patient with a histological diagnosis of PCNSL treated by Methotrexate and Methotrexate + GKRS r. The study received approval through the Institutional Review Board. Strict inclusion and exclusion criteria were employed (Table 1). All patient was included if they have a pathology report of histologically confirmed PCNSL Primary inclusion criteria was subjects received Methotrexate therapy as single agent in a standard protocol dosage of $8 \mathrm{~g} / \mathrm{m}^{2}$. This chart review evaluate clinical data and MRI images from June 1995 - May 2015.

Patients with diagnosis of PCNSL by patho-histology report of the brain lesion observed on MRI were added. The presence and size of the tumor, as well as its localization were confirmed using Brain MRI Neuro- Oncologist and Radio-Oncologist Protocol. The maximal brain tumor was measured at $1 \mathrm{~cm}$ and $2 \mathrm{~cm}$ proximal to the most distal aspect of its midline. Primary outcomes was survival rate and secondary assessed by the sensibility and decrease of the brain tumor lesion as noted on MRI.

\section{Chart review}

One experienced blinded post-doctoral fellow collected the data using red cap system from eligible patients. Those patients underwent Gamma Knife Radiosurgery and/or Methrotexate dose from institutional protocol. Patients' inclusion criteria are outlined in Table 1.

Subjects was excluded on various grounds including whether or not they previously failed a surgical brain tumor removal, radiation therapy or any other chemotherapy protocol.

\section{Statistical analysis}

A power analysis by mortality rate had been evaluate by y a priori sample size calculation performed on the basis of a prior randomized study [9], which evaluated using a survival rate with a sample size of 40 patients. This study suggested a sample size of at least 31 subjects per group to detect the minimal clinically important difference in mortality rate of 1.5 standard deviations $(\sigma)=1.5, \alpha=0.05, \beta=0.20$ ). Additionally, a general estimate for detecting a one-unit change on an ordinal scale of $136(\sigma=1.0, \alpha=0.05, \beta=0.20)$ resulted in the same number. The Wilcoxon rank-sum test was used for comparison of continuous data between groups. Differences between means were analyzed using two-sided t-tests. Comparison of categorical data was performed using Pearson chi-square or Fisher's exact probability test as indicated. Ordinal ranking scores were compared using the MantelHaenszel test. Differences less of $\mathrm{p}=0.05$ or less was reported statistical significant [10].

\section{Results}

Between June 1995 and May 2015, 128 charts was evaluated. 73 subjects in control group (Methrotezate) and 55 (Methrotexate + GKRS). Follow-up period 24 to 49 months (mean 36.9 months). There were no statistically significant differences in patient demographics or histology diagnosis (Table 2). Patients was treated using GKRS doses ranging from 12 Gy to 26 Gy (median:13.6 Gy) (Figure 1). Median survival was rate 26. 8 months in Control group and 47.6 in GKRS group from the initial diagnosis. All lesions showed a complete response after Radiosurgery when evaluated using magnetic resonance imaging 3-8 weeks (mean range 5.8 weeks).

The histologic finding in all patients was the presence of large, diffuse B-cell lymphoma. The symptoms and signs of the patients remained evidently improved within 2 - 6 weeks after GKRS and 4-10 weeks after chemotherapy alone. All treated lesions by GKRS showed complete response in magnetic resonance imaging 3 weeks to 8 weeks (mean range 6.3 weeks). There was a statistical significant difference of median survival rate chemo group alone 36.3 months from the initial diagnosis and 49.8 months in GKRS group $(\mathrm{P}=0.0034)$ [11,12]. The doses ranged from 12 Gy to $26 \mathrm{~Gy}$ (median: 13.8) $50 \%$ isodose line (range: 45-85; median 50) (Table 3). The clinical data was gathered for brain tumor lesion control, disease control, toxicity, recurrence and survival. The Kanofsky performance grade was enhanced from a preoperative average of $55 \%$ to a postoperative one of $88 \%$ in GK group. Significant factors contributing to survival greater 24 months following GKRS, were increased marginal dose (Odds ratio $=6.5$,

\section{INCLUSION CRITERIA}

Patient with diagnosis of PCNSL and treated by Methotrexate

Primary CNS Lymphoma diagnosed by Neuro Histology Pathologist

\section{EXCLUSION CRITERIA}

AIDS diagnosis at time of treatment and diffuse CNS lymphomas

Patient with addiction to illegal drugs, solvents, or alcohol who are currently using or previously attempted or failed a treatment program

Patients with bacteremia, systemic infection, or infection

Patients pregnant or nursing

History of previous brain surgery or brain tumor

History of systemic Disease

Table 1: Inclusion and exclusion criteria.

\begin{tabular}{|l|c|c|c|}
\hline Patient Parameters & Control & GKRS Group & P value \\
\hline Number of Patients & 73 & 55 & NA \\
\hline Age (years) & $58.1 \pm 5.3$ & $56.9 \pm 3.3$ & 0.685 \\
\hline Sex & $33(70 \%)$ & $26(56 \%)$ & 0.51 \\
\hline Male & $40(30 \%)$ & $19(44 \%)$ & \\
\hline Female & $24.1 \pm 2.6$ & $22.2 \pm 3.1$ & 0.39 \\
\hline Body-mass index $\left(\mathrm{kg} / \mathrm{m}^{2}\right)$ & & & \\
\hline Pre-op Diagnosis & 16 & 22 & 0.25 \\
\hline Tobacco use & $18.1 \pm 1.3$ & $19.1 \pm 0.9$ & 0.347 \\
\hline Pre-albumin (mg/dL) & $3.4 \pm 0.4$ & $3.2 \pm 0.4$ & $0.89^{\dagger}$ \\
\hline Albumin (g/dL) & $1745 \pm 468$ & $1798 \pm 401$ & 0.20 \\
\hline Absolute Lymphocyte & & & \\
\hline Count (cells/ML) & 26.8 months & 47.6 months & 0.0034 \\
\hline Median Survival & $(18.3-29.1)$ & $(28.3-49.1)$ & $0.59^{\dagger}$ \\
\hline Pre-op Diagnosis & & & \\
\hline
\end{tabular}

Table 2: Demographic and perioperative data.

\section{Mets / lymphoma}

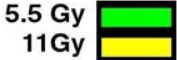

1st Row 2nd Row

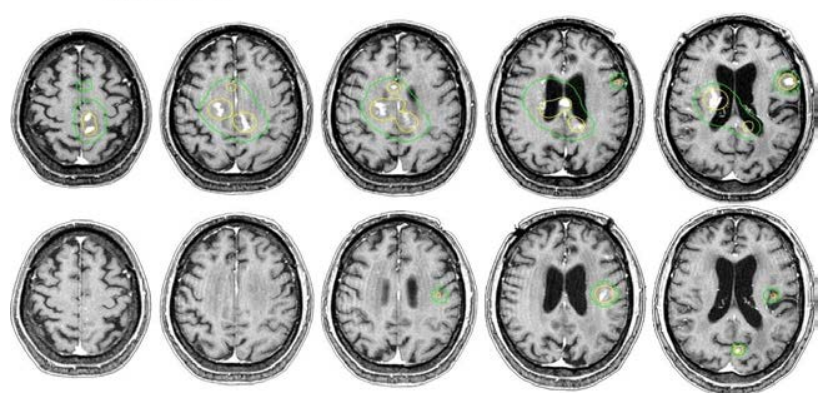

Figure 1: Brain MRI before and 2 months after GK treatment 


\begin{tabular}{|c|c|c|c|}
\hline Patient Parameters & Control Group & Gamma Knife & P Value \\
\hline $\begin{array}{l}\text { Tumor Size } \\
\text { Mean Size } \mathrm{cm}^{3} \\
\text { Median Size } \mathrm{cm}^{3} \\
\text { Size Range } \mathrm{cm}^{3}\end{array}$ & $\begin{array}{c}3.7 \\
2.9 \\
1.3-6.9\end{array}$ & $\begin{array}{c}3.5 \\
2.7 \\
1.5-6.4\end{array}$ & $\begin{array}{l}0.18 \\
0.27 \\
0.21\end{array}$ \\
\hline $\begin{array}{l}\text { Presence of single or multiple lesion } \\
(\%) \\
\text { Single } \\
\text { Multiple }\end{array}$ & $\begin{array}{l}18 \% \\
92 \%\end{array}$ & $\begin{array}{l}22 \% \\
78 \%\end{array}$ & $\begin{array}{l}0.35 \\
0.28\end{array}$ \\
\hline $\begin{array}{l}\text { Tumor location } \\
\text { Frontal } \\
\text { Parietal } \\
\text { Temporal } \\
\text { Occipital } \\
\text { Cerebellum }\end{array}$ & $\begin{array}{c}10 \\
14 \\
19 \\
7 \\
6\end{array}$ & $\begin{array}{c}12 \\
11 \\
14 \\
7 \\
4\end{array}$ & $\begin{array}{c}0.12 \\
0.106 \\
0.09 \\
0.58 \\
0.31\end{array}$ \\
\hline $\begin{array}{l}\text { Extra cranial metastasis } \\
\text { Yes } \\
\text { No }\end{array}$ & $\begin{array}{l}10 \\
63\end{array}$ & $\begin{array}{l}16 \\
39\end{array}$ & $\begin{array}{c}0.09 \\
0.072\end{array}$ \\
\hline $\begin{array}{l}\text { VP Shunt } \\
\text { Yes } \\
\text { No }\end{array}$ & $\begin{array}{l}24 \\
49\end{array}$ & $\begin{array}{l}14 \\
41\end{array}$ & $\begin{array}{l}0.068 \\
0.35\end{array}$ \\
\hline Prior cancer & 11 & 6 & 0.24 \\
\hline Systemic symptoms & 5 & 3 & 0.587 \\
\hline Positive CSF cytology examination & 73 & 51 & 0.187 \\
\hline High CSF protein level & 51 & 43 & 0.14 \\
\hline Multiple lesions & 42 & 29 & 0.0687 \\
\hline \multicolumn{4}{|c|}{$\begin{array}{l}\text { Values are given as mean and standard deviation, } \\
\text { with or without range in parentheses and } 95 \% \\
\text { confidence interval in brackets. }\end{array}$} \\
\hline $\begin{array}{l}\text { †Relationship between the number } \\
\text { and the total number of assessed pe }\end{array}$ & $\begin{array}{l}\text { positive cases } \\
\text { nts. }\end{array}$ & & \\
\hline
\end{tabular}

Table 3: Brain tumor related outcomes.

$\mathrm{p}=0.031,95 \% \mathrm{CI}(1.4-18.57)$, increase in maximal dose (Odds ratio $=1.54, \mathrm{p}=0.012,95 \% \mathrm{CI}(1.15-2.89)$, and increase in pre-treatment Karnofsky score (Odds ratio $=5.13, \mathrm{p}=0.008,95 \%$ CI $(1.55-85.1)$. The side effects attributed to GKRS were minimal; none of the patients had a deterioration related to the treatment. No complications were related after procedure $[13,14]$

\section{Discussion}

In the past 30 years, radiotherapy was the primary therapeutic modality used to manage patients with PCNSL, even though the addition of chemotherapy has not substantially enhanced their effect [4]. The side effects of intrathecal methotrexate, presumptuous preliminary comorbidities and the size of the brain lesions are risk factors to be evaluated. Combined chemo and radiotherapy, radiotherapy alone and variable chemotherapy regimens and radiation doses, as well as straight chemo and radiotherapy might prove to be more harmful than beneficial $[5,6]$. In fact, radiotherapy has been linked to severe neurotoxicity, predominantly amongst elder patients [7-9]. High incidence of neurotoxicity has been reported in patients receiving radiotherapy, mostly in patients older than 65 years. Moreover, up to $35 \%$ of patients present with neurologic deterioration after radiotherapy treatment, resulting in a mortality rate of $30 \%$ in 5 years. Consequently, the dilemma postured by PCNSL treatment is determination of a superior approach that would offer higher success rates while limiting adverse effects such as neurotoxicity. Importantly, not a single patient experienced any mental or memory impairment in our study, which is in partial agreement with an earlier report. Kenia, et al. [10], described a mean recurrence-free period of 24.4 months for their patients. In our observational study 16 of the patients have more than 7 brain lesions with poor clinical prognoses. 9 patients developed new lesions in other areas of the brain and repeated GKS was performed. No local recurrence was observed in any patient at a mean of 30.6 months post-GKS treatment. Our findings are in accordance with those of Kenia, and seems to be at a higher success rate that those described by others in treating patients with chemotherapy and/ or radiotherapy alone. There appeared to be a predisposition in the direction of new tumor growth in individuals who did not undergo GK treatment. We establish no suggestion of a connection between recurrence and original appearance of multiple or solitary lesions but current systemic chemotherapy would be essential. The prognosis of untreated PCNSL remains grim with median survival time being low. GKRS appears to be a valuable method for the management of primary and relapsed CNSL, since it enables exceptional local control in a shortterm management period without severe complications.

\section{Limitation}

This study has a few limitations including: (i) it is a retrospective, comparative study and (ii) lack of prospective, randomized data collection confines us in assessing the full benefits and complications of GKRS.

\section{Conclusion}

Given the well tumor control, enhanced overall survival period and lesser number of complications, GKRS can be an ideal co-adjuvant treatment option for the patients with PCNS lymphoma adding of standard Methrotexate treatment. In addition, well and prompt tumor was reported using Gamma Knife Radiosurgery allowing the immediate improve of neurological symptoms.

\section{Acknowledgements}

The authors would like to express their appreciation to Marcos E SanchezGonzales MD, Phd, Juan D. Oms, MD and Sandy Sosa-Guerrero RN.

\section{References}

1. Ostrom QT, Gittleman H, Farah P, Ondracek A, Chen Y, et al. (2013) CBTRUS statistical report: Primary brain and central nervous system tumors diagnosed in the United States in 2006-2010. Neuro Oncol 15 Suppl 2: ii1-56.

2. Mahadevan A, Rao CR, Shanmugham M, Shankar SK (2015) Primary centra nervous system diffuse large B-cell lymphoma in the immunocompetent: Immunophenotypic subtypes and Epstein-Barr virus association. J Neurosci Rural Pract 6: 8-14.

3. Weller M, Martus $P$, Roth $P$, Thiel E, Korfel A, et al. (2012) Surgery for primary CNS lymphoma? Challenging a paradigm. Neuro Oncol 14: 1481-1484

4. Nakajima S, Okada T, Yamamoto A, Kanagaki M, Fushimi Y, et al. (2014) Primary central nervous system lymphoma and glioblastoma: differentiation using dynamic susceptibility-contrast perfusion-weighted imaging, diffusionweighted imaging, and (18) F-fluorodeoxyglucose positron emission tomography. Clin Imaging 39: 390-395.

5. Tun HW, Personett D, Baskerville KA, Menke DM, Jaeckle KA, et al. (2008) Pathway analysis of primary central nervous system lymphoma. Blood 111 3200-3210.

6. Liu HL, Liu T, Ma C, Zhao Y, Zhou Y (2015) [Clinical characteristics and prognosis of 20 cases of primary central nervous system lymphoma]. Zhongguo Shi Yan Xue Ye Xue Za Zhi 23: 111-118.

7. Thiel E, Korfel A, Martus P, Kanz L, Griesinger F, et al. (2010) High-dose methotrexate with or without whole brain radiotherapy for primary CNS lymphoma (G-PCNSL-SG-1): a phase, randomised, non-inferiority trial. Lancet Oncol 11: 1036-1047.

8. Rubenstein JL, Hsi ED, Johnson JL, Jung SH, Nakashima MO, et al. (2013) Intensive chemotherapy and immunotherapy in patients with newly diagnosed primary CNS lymphoma: CALGB 50202 (Alliance 50202). J Clin Oncol 31: 3061-3068.

9. Wang CC, Carnevale J, Rubenstein JL (2014) Progress in central nervous system lymphomas. Br J Haematol 166: 311-325. 
Citation: Alvarez AM, Valerio JE, Coy S, Alan A Stein BS, Wolf A (2015) Primary cns Lymphoma: Analysis of Treatment by Gamma- Knife Radiosurgery and Chemotherapy in a Prospective, Observational Study. J Integr Oncol 4: 150. doi:10.4172/2329-6771.1000150

10. Kenai H, Yamashita M, Nakamura T, Asano T, Momii Y, et al. (2006) Gamma Knife surgery for primary central nervous system lymphoma: usefulness as palliative local tumor control. J Neurosurg 105 Suppl: 133-138.

11. Aoki H, Ogura R, Tsukamoto Y, Okada M, Natsumeda M, et al. (2013) Advantages of dose-dense methotrexate protocol for primary central nervous system lymphoma: comparison of two different protocols at a single institution. Neurol Med Chir 53: 797-804.

12. Matsumoto $Y$, Horiike S, Fujimoto $Y$, Shimizu D, Kudo-Nakata $Y$, et al. (2007) Effectiveness and limitation of gamma knife radiosurgery for relapsed central nervous system lymphoma: a retrospective analysis in one institution. Int $\mathrm{J}$ Hematol 85: 333-337.

13. Levin N, Soffer D, Grissaru S, Aizikovich N, Gomori JM, et al. (2008) Primary T-cell CNS lymphoma presenting with leptomeningeal spread and neurolymphomatosis. J Neurooncol 90: 77-83.

14. Beiko J, Suki D, Hess KR, Fox BD, Cheung V, et al. (2014) IDH1 mutant malignant astrocytomas are more amenable to surgical resection and have a survival benefit associated with maximal surgical resection. Neuro Oncol. 16 81-91. 